\title{
Naltrexone a potential therapeutic candidate for COVID-19
}

\author{
Abhinav Choubey ${ }^{1}$, Budheswar Dehury ${ }^{2}$, Sunil Kumar ${ }^{3}$, Bikash Medhi $^{4}$, and Prosenjit \\ Mondal $^{1}$ \\ ${ }^{1}$ IIT Mandi \\ ${ }^{2} \mathrm{KiiT}$ \\ ${ }^{3}$ ICAR-Indian Agricultural Statistical Research Institute \\ ${ }^{4}$ PGIMER
}

June 10, 2020

\begin{abstract}
Severe acute respiratory syndrome coronavirus 2 (SARS-COV-2) has recently emerged as a new public health threat. SARSCoV-2 is the cause of coronavirus disease (COVID-19) that has resulted in a global pandemic. At the time of writing, approximately 6.6 million cases have been reported worldwide. Like other coronaviruses, SARS-CoV-2 relies on the surface Spike glycoprotein to access the host cells, mainly through the interaction of its Receptor Binding Domain (RBD) with the host receptor Angiotensin-Converting Enzyme2 (ACE2). SARS-CoV-2 infection induces a profound downstream proinflammatory cytokine storm. This release of the proinflammatory cytokines is underpinning lung tissue damage, respiratory failure, and eventually multiple organ failure in COVID-19 patients. The phosphorylation status of ERK1/2 is positively correlated with virus load and ERK1/2 inhibition suppressed viral replication and viral infectivity.Therefore, molecular entities able to interfere with binding of the SARS-CoV-2 Spike protein to ACE2, or damping hyperinflammatory cytokines storm, blocking ERK1/2 phosphorylation have a great potential to inhibit viral entry along with viral infectivity. Herein, we report that the FDAapproved non-peptide opioid antagonist drug, naltrexone suppresses high fat/LPS induced pro-inflammatory cytokine release both from macrophage cells and Adipose Tissue Macrophage (ATM). Moreover, Low Dose Naltrexone (LDN) also showed its activity as an ERK1/2 inhibitor. Notably, virtual docking and simulation data also suggest LDN may disrupt the interaction of ACE2 with RBD and thus LDN may be considered as a target as the treatment and (or) adjuvant therapy for coronavirus infection.
\end{abstract}

\section{Introduction}

Coronavirus disease 2019 (COVID-19) is a major health concern, clinical symptoms of the disease vary from a mild illness, acute respiratory issues to multi-organ failure ${ }^{1,2}$. Older age, diabetes, cardiac diseases predict poor prognosis in COVID-19 patients ${ }^{3,4}$. Although much is known about the mortality of the COVID-19, however, details of the cellular responses to this virus are not known.

Several preclinical and clinical trials data have indicated an elevated cytokine/chemokine response in severe COVID-19 patients and identifies cytokine storm as the most potentially dangerous event for mortality ${ }^{5-7}$. Several kinases in the MAPK/ERK (mitogen-activated protein kinases/extracellular signal-regulated kinases) pathway are essential for viral replication. ERK1 and ERK2 phosphorylate HIV-1 proteins and enhance viral infectivity $^{8}$. The phosphorylation status of ERK1/2 is positively correlated with virus load and ERK1/2 inhibition suppressed viral replication and viral infectivity ${ }^{8}$. Some preclinical findings have suggested targeting ERK1/2 pathway to halt the viral replication and severity of SARS-COV $-2^{9}$.

SARS-CoV-2 uses the homotrimeric spike glycoprotein as the main protein that interacts with the host by binding to host cell receptors (ACE2) to mediate virus invasion for cell entry. Some recent studies 
have highlighted the important role of ACE2 in mediating entry of SARS-CoV-2 ${ }^{10,11}$. A recent report also recognized several critical residues in RBD, including its receptor-binding motif (RBM) that directly contacts with human ACE2 ${ }^{12}$. In vitro study using HeLa cells also reinforced the role of ACE2 in mediating entry of SARS-CoV-2, where HeLA cells expressing ACE2 are susceptible to SARS-CoV-2 infection whereas those without ACE2 are not. Furthermore, In vitro binding measurements, experiments showed that the SARS-CoV-2 RBD binds to ACE2 with an affinity in the nanomolar range, indicating that the RBD is a key functional component that is responsible for the binding of SARS-CoV-2 by ACE2 and can be considered as a target for the treatment of coronavirus infection to block SARS-CoV-2 from entering host cells ${ }^{11,12}$.

There is a very fast ongoing search for therapeutics acting on SARS-CoV-2. Depending on the activity, the therapies can be divided into majorly main categories: (1) Inhibiting the viral RNA synthesis and replication, (2) Deterring the virus from binding to human cell ACE2 receptors, (3) Reinstating the innate immunity, and (4) Blocking the host's specific receptors or enzymes. Despite many experimental and computational studies currently exploring all of these categories, to date, there is no confirmed effective treatment specifically available for COVID-19.

In this study, we report that the FDA-approved non-peptide opioid antagonist drug ${ }^{13}$, LDN suppresses high fat/LPS induced pro-inflammatory cytokine release both from macrophage cells and Adipose tissue macrophage (ATM). LDN also showed activity as an ERK1/2 inhibitor. Moreover, virtual docking and simulation data also suggest LDN may disrupt the interaction of ACE2 with RBD. As a reliable COVID-19 vaccine is unlikely to available before the maximal infection of COVID-19 has occurred, it is essential to establish therapeutics for the COVID-19 patients, based on our data, we proposed FDA-approved LDN can be used in combination or as an adjuvants therapy to treat mild to moderate symptomatic COVID-19 patients

\section{Research Design and Methods:}

Drugs and Chemicals: A pharmacological low dose of naltrexone hydrochloride (LDN) obtained from MP Biomedicals (151725) was used.

Cell lines and culture treatment: Murine macrophage (Raw264.7) cell line was cultured in RPMI media supplemented with $10 \%$ fetal bovine serum and $1 \%$ penicillin-streptomycin

Cell viability: Cell viability assay was carried out in Raw264.7 cells using MTT dye (3-(4, 5-dimethyl thiazol-2yl)-2, 5-diphenyl tetrazolium bromide) as reported earlier ${ }^{32}$. Cells were seeded in a 96-well plate and allowed to grow overnight. Further cells were treated with varying doses $(0,2,4,6,8,10,20,40 \mu \mathrm{M})$ of the LDN for $24 \mathrm{hrs}$. Post-treatment, $10 \mu \mathrm{l}$ of MTT $(5 \mathrm{mg} / \mathrm{ml}$ stock in PBS) was added to all the wells. The formazan crystals thus formed were solubilized in 200 $\mathrm{\mu l}$ DMSO and the absorbance was recorded using (Infinite M200 Pro TECAN).

RNA isolation and gene expression profile: Raw264.7 cultured cells were treated with $5 \mu \mathrm{M}$ of LDN in the presence and absence of LPS. RNA was isolated from the cells using RNA-Xpress reagent (HIMEDIAMB601) and $1 \mu \mathrm{g}$ of RNA was reverse-transcribed for quantitative RT-PCR analysis. The results were normalized using housekeeping genes $18 \mathrm{~S}$ (rRNA) and expression was calculated as fold change by normalizing the control values. Primers are provided in supplemental experimental procedures (table S2).

Western Blot Analysis: Raw264.7 cultured cells were incubated with LDN (0 and $5 \mu \mathrm{M})$ in the presence or absence of LPS. After treatment, cells were lysed in RIPA buffer containing 1\% protease- phosphatase inhibitors. Protein concentration was determined by BCA assay reagent as described in the manufacturer's (Thermo Scientific-23227) manual. Protein was loaded on SDS-PAGE and electro-blotted on to PVDF membranes. The membrane was incubated in $5 \%$ milk blocking solution for $2 \mathrm{hrs}$ at room temperature (RT) and probed against primary antibody (1:2000 diluted in TBST; After washing with TBST, the membrane was incubated with HRP conjugated IgG secondary antibody for $2 \mathrm{hrs}$ and visualized by chemiluminescence. A list of antibodies is provided in supplemental experimental procedures (table S2).

ATM Isolation and Analysis: Mice were euthanized chemically and epididymal fat was processed for 
isolation of ATM. 1 gram of adipose fat was rinsed in PBS and minced to small pieces in HEPES-DMEM buffer containing $10 \mathrm{mg} / \mathrm{ml}$ BSA. The suspension was centrifuged at $1,000 \mathrm{~g}$ for $10 \mathrm{~min}$ and the resultant supernatant was pipette off to fresh tubes. $1 \mathrm{mg} / \mathrm{ml}$ of collagenase type- IV and $50 \mathrm{U} / \mathrm{ml}$ DNAse-II were added to this suspension and incubated at $37^{\circ} \mathrm{C}$ for 45 mins with moderate shaking, filtered through 250-micron filter and the resultant solution was centrifuged again at 1,000g for 10 mins. Floating cells contained adipocyte and pellet are SVC. RBC lysis buffer was added gently to disrupt the sedimented pellets and centrifuged at 1,000 $\mathrm{g}$ for $10 \mathrm{~min}$ at $4^{\circ} \mathrm{C}$. Fat macrophage cells were isolated by using BD IMag anti-mouse CD11b+ magnetic beads through positive selection under the magnetic field. The percentage purity of macrophage isolation was determined by FACSCANTO II flow cytometer using APC tagged CD11b monoclonal antibody. The isolated macrophage was processed for RNA isolation, cDNA synthesis and various M1-M2 markers were evaluated using real-time PCR.

\section{Structure preparation and molecular docking:}

The experimentally solved SARS CoV-2 RBD-ACE2 complex (PDB ID: 6M0J solved at $2.45 \AA$ ) was obtained from $\mathrm{PDB}^{12}$. The ligand naltrexone (PubChem CID: 5360515) was extracted from the PubChem database. An attempt was made to dock to explore the binding mode of naltrexone onto the binding interface of the RBD-ACE2 complex using AutoDock version 4.2 $2^{33}$ and AUTODOCK tools 1.5.6. Before docking, the protein was prepared by the removal of small molecules and waters. Then, polar-hydrogen atoms were added to the structure followed by Gasteiger charges calculation. Ligand centered map was generated with a spacing of $0.375 \AA$ and grid dimensions of $46 \times 46 \times 46 \mathrm{~A}^{3}$ (x-y-z) covering the biding interfaces residues (which includes the receptor-binding motif viz., RBM) of the complex (coordinates of central grid point of maps:-34.512, $20.978,4.521)$. Default settings were used for all other parameters while performing docking with the number of GA run to 100. From the resultant docked conformations, the top-ranked conformation with the least free energy of binding, hydrogen-bonding and interatomic-bonding pattern was selected for further optimization by employing long term MD simulation. PyMOL (The PyMOL Molecular Graphics System, Version 2.0 Schrodinger, LLC.) and BIOVIA Discovery Studio Visualizer version4.5 were employed used to visualize the inter-molecular contacts between naltrexone with RBD-ACE2 complex.

\section{Molecular dynamics simulation:}

To study the dynamic behavior, stability, and conformational flexibility RBD-ACE2- naltrexone complex, all-atoms MD simulations were performed as reported previously ${ }^{17,34,35}$. CHARMM36 force fields were used for topology building of protein in GROMACSv2019.4 package $^{36}$. The ligand topology was derived from CHARMM General Force Field (https://cgenff.umaryland.edu/) $)^{37}$. The structure was solvated in a cubic water box in TIP3P water model. The system charge was electro-neutralized by adding $0.15 \mathrm{M} \mathrm{NaCl}$ to the solvated system. To eliminate bad contacts in the complex system energy minimization was done with the steepest descent algorithm in 5,000 steps. The non-hydrogen atoms of the ligands were restrained, and system equilibration was done in two steps including NVT and NPT ensembles in $10 \mathrm{~ns}$ at $300 \mathrm{~K}$ in atmospheric condition (1 atm). Finally, production MD was performed $100 \mathrm{~ns}$ at $300 \mathrm{~K}$ with a 2 -femtosecond (fs) time step using Leapfrog integrator. The resultant trajectory was explored to understand the structural dynamics of the complex system through various utility toolkits of GROMACS. Stability parameters including backbone root mean square deviation (RMSD), the radius of gyration (Rg), C $\alpha$-root mean squared fluctuations (RMSF), and intermolecular hydrogen bond (H-bond) distributions were computed for the complex system. 2D graphs were plotted using XMGrace, while, interaction images were plotted using BIVIA DSV and PyMOL.

Statistical analysis: All the data presented is as mean \pm SEM of three individual experiments unless specified. Comparisons between means were performed using Student $t$-test for unpaired data within two conditions.

\section{Results:}

\section{LDN diminished LPS induced cytokine storm.}

Cytokine storm is a very commonly observed factor in most severe COVI19 patients and also one of the 
leading causes of mortality ${ }^{5-7}$. Peripheral blood of severe COVID-19 patients has also shown a high level of cytokine storm ${ }^{14}$. Keeping in mind the ability of lipopolysaccharide (LPS) to cause sepsis and triggers an uncontrolled systemic inflammatory response in murine macrophage cells ${ }^{15}$, we treated macrophage cells with LPS $(1 \mu \mathrm{g} / \mathrm{ml})$ in presence and absence of LDN $(5 \mu \mathrm{M})$. Results demonstrated that LPS treatment significantly induced expression on pro-inflammatory cytokines (IL-1 $\beta$, IL-6and TNF-a) whereas, LDN significantly inhibited LPS expression of IL-1 $\beta$, IL-6and TNF-a in macrophage cells (Fig. 1A ). Adipose tissue macrophage (ATM) is closely linked to this inflammatory condition which leads to numbers of diseases and the ability of HFD feeding on increased LPS uptake and trafficking to macrophages and other targets are well known ${ }^{16}$. Hence in this study, we investigated the effect of High Fat Diet (HFD) on cytokines expression in purified ATMs. Expression of pro-inflammatory markers such as $I \Lambda-1 \beta, T N F$-a, and IL6 was induced (Fig-1B ) whereas LDN attenuates HFD induced expression profile of pro-inflammatory cytokine. LDN shows a protective (by reducing elevated M1 cytokines) from HFD mediated inflammation. Next, we tested the possible involvement of High Fat Diet (HFD) in inducing the release of proinflammatory cytokines, and we also determine the effects of LDN on release of proinflammatory mediators in HFD mice model (Fig 1C ). Serum from HFD mice show a significantly enhanced release proinflammatory mediators (IL-1B, TNF- $\alpha$ and IL-6) and interestingly we found that LDN treatment attenuated HFD induced serum IL-1B, TNF- $\alpha$ and IL-6 level (Fig-1C ). Cell viability was determined by utilizing MTT assay in macrophage cell line. The assay revealed that the selected range of dose is safe and did not affect cell viability at the different doses exposed (Fig S1). These data suggest that HFD diet induced mice persuaded the release of various proinflammatory mediators, LDN treatment can significantly abrogate HFD induced release of proinflammatory mediators in serum.

\section{LDN down-regulation of LPS induced ERK1/2 phosphorylation.}

ERK1 and ERK2 mitogen-activated protein kinases (MAPK) play a critical role in the regulation of cell proliferation and differentiation in response to mitogens and other extracellular stimuli. Mitogens and cytokines that activate MAPK in cells have been shown to activate virus replication ${ }^{8}$. The cytokine storm is a wellknown factor that is increasing the severity and mortality in COVID $19^{5-7}$. Moreover, the phosphorylation status of ERK1/2 is positively correlated with virus load and reduced ERK1/2 phosphorylation suppressed viral replication significantly, thus reduced viral $\operatorname{load}^{8}$. We pre-treated the cells with LDN and exposed to LPS for 18 hours. As shown in Fig.1D . the phosphorylation of ERK 1/2 was increased after LPS exposure which was significantly suppressed by LDN treatment (Fig 1D). This finding suggests that LDN can acts as an inhibitor for ERK1/2 activation and may reduce the infectivity of virions.

In silico studies revealed LDN interacts with the receptor-binding motif of SARS-CoV-2 -RBD

The latest research shows that the spike-receptor binding domain (RBD) sequence of SARS-CoV-2 interacts with host receptor ACE2 and this RBD-ACE2 complex plays a key role in virus invasion and virulence. Based on the current research progress, the RBD-ACE2 complex is considered as a target for the treatment of coronavirus infection to block SARS-CoV-2 from entering host cells.

To understand the mode of interaction naltrexone in the binding interface of RBD-ACE2 complex, molecular docking was performed using AutoDock. The docking scores of the top ten complexes have been summarized in Table S1. As evidenced by the top-ranked conformation (as shown in Figure 2 ), the naltrexone prefers to bind in the cavity formed RBD and ACE2 receptor. Tyr505 and Glu406 of RBD formed two crucial hydrogen bonds with the naltrexone with an atomic distance of 2.08 and 1.80, while, Arg 403 formed electrostatic contact. While the His34, Glu37, and Phe390 of ACE2 displayed several hydrophobic contacts (mostly pi-alkyl contacts) with naltrexone.

\section{Trajectory analysis.}

The dynamics stability of the RBD-ACE2-naltrexone complex was analyzed by performing all-atoms MD simulations of 100ns in GROMACS. The backbone RMSD analysis provides important information on the stability of protein and protein-ligand complexes and the time when simulation reached equilibrium. The RMSD of the RBD-ACE2-naltrexone complex displayed an average RMSD $\sim 2.46 \mathrm{~nm}$ throughout the entire 
simulation (Figure 3A ). Besides, the RMSD of ligand was also found to be stable with very minimal deviation as compared to the starting conformation. Overall, the complex system displayed the least backbone deviation, indicates that docked conformation is accurate and remained stable over the $100 \mathrm{~ns}$ timescale. Radiuses of gyration assess the compactness of the system, where a compact gyradius of $\sim 3.24 \mathrm{~nm}$ for the complex indicates the consistent shape and size of the system during the simulation (Figure 3B ). The residue flexibility of protease and RBD-ACE2 structures were examined by performing C $\alpha$ RMSF analysis of both the sub-units (Figure ). The average RMSF of ACE2 was found to be $0.14 \mathrm{~nm}$, while for the RBD it was reported to be $0.17 \mathrm{~nm}$ (for the receptor-binding motif $\sim 0.16 \mathrm{~nm}$ ). The receptor-binding motif of RBD displayed a high degree of flexibility and the residues participated in the ligand interaction also portrayed higher RMSF indicating their participation in ligand recognition.

The intermolecular hydrogen bonds (H-bonds) between interacting atom pairs in a protein-ligand complex plays a vital role in the stability and molecular recognition process ${ }^{17}$. The intermolecular H-bonds were calculated with respect to time during the $100 \mathrm{~ns}$ MD simulation to see the dynamics stability RBD-ACE2naltrexone complex (Figure 4A ). Though we observed an increased differential H-bonding during the initial $20 \mathrm{~ns}$ equilibration phase, however a stable trend with an average of $\sim 4.13 \mathrm{H}$-bonds are noticed from $60-100$ ns. Close inspection of snapshots from MD revealed that some of the H-bonds were broken out during MD simulation, but at a later stage they well rewarded by new H-bonds, and hydrophobic contacts. This may be due to the structural re-orientation of ligand naltrexone in the binding pocket. The structural superposition of the docked complex with the cluster representative obtained from clustering analysis displayed C $\alpha$ RMSD of $0.65 \AA$ indicated that complex retained its structural integrity throughout the simulation (Figure 4B ). However, close observation of the ligand for the initial starting structure used MD revealed that the ligand tends to reorient within the binding site during MD (as shown in Figure 3C) but form a close tight network of hydrogen bonds and non-bonded contact with ACE and RBM of RBD. Analysis of the cluster representative revealed the crucial residues of RBD and ACE2 involved in the crucial interaction with naltrexone. Lys417 and Asp405 from RBD formed two hydrogen bonds with naltrexone, while Glu37 of ACE2-formed the lone hydrogen bond(Figure S2 ). Many electrostatic and hydrophobic contacts were also observed in the complex (Figure S2 ) where, Ile418, Gln409, and Tyr505 from RBD consistently formed close contact with ligand indicates their strong participation in the interaction mediated by naltrexone.

Discussion:

As a reliable COVID-19 vaccine is unlikely to available before the maximal infection of COVID-19 has occurred, it is essential to establish therapeutics for the individuals at moderate and high risk of the disease. So far, anti-viral medication is a major available option for COVID19 patients. LDN which showed properties like anti-inflammatory, ERK1/2 inhibitory, and as well virtual docking and simulation data also suggested LDN may disrupt the interaction of ACE2 with RBD. LDN has been gaining credibility in its ability to halt the progression of several diseases without significant side effects when administered in low dosage $\mathrm{e}^{18-22}$.

Spike is the main structural protein of coronavirus and assembles into a special corolla structure on the surface of the virus as a trimer. Spike is the main protein that interacts with the host by binding to host cell receptors to mediate virus invasion. Spike is cleaved into S1 and S2 by the host cell protease like TMPRSS2, etc. The main function of S1 is to bind with host cell surface receptor; ACE2 and the S2 subunit mediates virus-cell and cell-cell membrane fusion ${ }^{11}$. Spike structural integrity and cleavage activation play a key role in virus invasion and virulence ${ }^{11,23}$. Therapeutic strategies to block coronavirus from entering host cells by targeting Spike proteins or specific receptors (ACE2) on the host surface are valuable for the development of anti-viral drugs. It is anticipated that potential ACE2 inhibitors may not be suitable to use as drugs for treating SARS-CoV-2 infection because the poor prognosis would be induced by the inhibition of ACE2 enzyme activities, considering ACE2 is a protective role against lung injury. Recently, the crystal structure of the receptor-binding domain (RBD) of the spike protein of SARS-CoV-2 bound to the cell receptor ACE2 has reported ${ }^{12}$. Lan et al mentioned there are 13 hydrogen bonds at the SARS-CoV-2 RBD-ACE2 interface, this involves multiple tyrosine residues (Tyr436, Tyr449, Tyr489, and Tyr505) from the SARS-CoV-2 RBD to form hydrogen-bonding interactions with the polar hydroxyl group ${ }^{12}$. 
The latest research further strengthened that the spike-RBD sequence of SARS-CoV-2 interacts with host receptor ACE2 and this RBD-ACE2 complex plays a key role in virus invasion and virulence ${ }^{11}$. Based on virtual screening results, LDN interacts with ACE2. Naltrexone prefers to bind in the cavity formed SARSCoV-2 RBD and ACE2 receptor. Tyr505 and Glu406 of RBD formed two crucial hydrogen bonds with the naltrexone with an atomic distance of 2.08 and 1.80, while, Arg403 formed electrostatic contact. While the His34, Glu37, and Phe390 of ACE2 displayed some hydrophobic contacts (mostly pi-alkyl contacts) with naltrexone. Overall this suggests LDN can strongly interact with SARS-CoV-2 RBD, including its RBM may further influence RDB- ACE2 binding, and host cell infectivity.

Therapeutic options for severe COVID-19 remain limited to date, Immunomodulatory agents that directly target the crucial cytokines involved in COVID-19 may also help in alleviating hyperinflammation symptoms, mild and severe cases in particular ${ }^{24}$. Corticosteroids are among the most commonly used drugs for immunomodulatory therapy of infectious diseases. However, the use of corticosteroids in the treatment of COVID-19 can cause host immune suppression and may delay viral clearance ${ }^{25}$. Combination of antibiotic, antiviral and steroid therapy exhibited respiratory failure and required non-invasive ventilation ${ }^{26}$. Elevated plasma IL-6 levels have been reported and to be predictive of a fatal outcome in COVID-19 patients ${ }^{527}$. Other than corticosteroids, Tocilizumab, a specific monoclonal antibody that blocks IL-6, has been recommended for use in severe or critically ill patients. Tocilizumab specifically binds to IL-6 receptor, and block its signalling cascade $^{28}$. However, clinical experience with tocilizumab in viral disease is very limited. Moreover, high costs and safety risks may be a barrier for the wide use of tocilizumab in the treatment of COVID-19.

Unlike high doses naltrexone, Low Dose Naltrexone (LDN; dose between 1 to $5 \mathrm{mg}$ ) has several mechanisms of action reported in the literature ${ }^{29}$. LDN stimulates the release of $\beta$-endorphins by acting on opioid receptor $^{29,30}$. LDN acts as a TLR4 antagonist, In human pilot study (4.5 $\mathrm{mg}$ of LDN daily) significantly reduced serum proinflammatory cytokines (IL)-1, IL-2, IL-12, IL-18, TNF-a etc ${ }^{31}$. Importantly, Low Cost, Low side effects, no reports of LDN interactions with other medications, and oral availability make LDN as a lucrative option to be used as immunomodulatory agent and may be considered for use in combination with antiviral drugs for the treatment of severe or critical cases of COVID-19.

Our data provide a proof-of-concept for the potential feasibility of repurposing of FDA approved non-peptide opioid antagonist; naltrexone as host-targeted broad-spectrum antiviral therapies to combat COVID-19 infections. The next step will be to confirm data in COVID-19 patients. LDN alone or as an adjuvant therapy with an antiviral agent may give physicians more time to provide supportive treatment for patients with COVID-19.

\section{Author contributions:}

$\mathrm{AC}$ conducted the majority of the experiments and analyzed the data. BD and SK conducted in silico studies. PM, designed the study and supervised the project. PM and AC, wrote the manuscript with support from BD ,SK and BM. PM is the guarantor of this work and, as such, has full access to all the data in the study and takes responsibility for the integrity of the data and the accuracy of the data analysis

Competing INTERESTS: authors declare no competing financial interest"

\section{Acknowledgments:}

We sincerely thank the BioX Centre of IIT Mandi for use of different analytical instruments AC thank Department of Science \& Technology (DST) INSPIRE fellowship, for his research fellowship. The authors received no funding from an external source.

\section{References:}

1. Zaim, S., Chong, J. H., Sankaranarayanan, V. \& Harky, A. COVID-19 and Multiorgan Response. Curr. Probl. Cardiol. 100618 (2020). doi:10.1016/j.cpcardiol.2020.100618

2. Wang, T. et al. Comorbidities and multi-organ injuries in the treatment of COVID-19. The Lancet 395 , e52 (2020). 
3. Fang, L., Karakiulakis, G. \& Roth, M. Are patients with hypertension and diabetes mellitus at increased risk for COVID-19 infection? The Lancet Respiratory Medicine 8 , e21 (2020).

4. Madjid, M., Safavi-Naeini, P., Solomon, S. D. \& Vardeny, O. Potential Effects of Coronaviruses on the Cardiovascular System: A Review. JAMA Cardiology (2020). doi:10.1001/jamacardio.2020.1286

5. Coperchini, F., Chiovato, L., Croce, L., Magri, F. \& Rotondi, M. The cytokine storm in COVID-19: An overview of the involvement of the chemokine/chemokine-receptor system. Cytokine Growth Factor Rev.(2020). doi:10.1016/j.cytogfr.2020.05.003

6. Rahmati, M. \& Moosavi, M. A. Letter to the Editor Cytokine-Targeted Therapy in Severely ill COVID-19 Patients : Options and Cautions.Eurasian J. Med. Oncol. 4, 179-180 (2020).

7. Zhang, W. et al. The use of anti-inflammatory drugs in the treatment of people with severe coronavirus disease 2019 (COVID-19): The experience of clinical immunologists from China. Clinical Immunology 214 , (2020).

8. Cai, Y., Liu, Y. \& Zhang, X. Suppression of Coronavirus Replication by Inhibition of the MEK Signaling Pathway. J. Virol.81, 446-456 (2007).

9. Mizutani, T. Signaling pathways of SARS-CoV in vitro and in vivo. inMolecular Biology of the SARSCoronavirus 305-322 (Springer Berlin Heidelberg, 2010). doi:10.1007/978-3-642-03683-5_19

10. Hoffmann, M. et al. SARS-CoV-2 Cell Entry Depends on ACE2 and TMPRSS2 and Is Blocked by a Clinically Proven Protease Inhibitor.Cell 181, 271-280.e8 (2020).

11. Walls, A. C. et al. Structure, Function, and Antigenicity of the SARS-CoV-2 Spike Glycoprotein. Cell $181,281-292 . e 6$ (2020).

12. Lan, J. et al. Structure of the SARS-CoV-2 spike receptor-binding domain bound to the ACE2 receptor. Nature581 , 215-220 (2020).

13. Vickers, A. P. \& Jolly, A. Naltrexone and problems in pain management. British Medical Journal 332 , $132-133$ (2006).

14. Wu, D. \& Yang, X. O. TH17 responses in cytokine storm of COVID-19: An emerging target of JAK2 inhibitor Fedratinib. J. Microbiol. Immunol. Infect. (2020). doi:10.1016/j.jmii.2020.03.005

15. Ramos-Benitez, M. J. et al. Fh15 Blocks the Lipopolysaccharide-Induced Cytokine Storm While Modulating Peritoneal Macrophage Migration and CD38 Expression within Spleen Macrophages in a Mouse Model of Septic Shock. mSphere 3 , (2018).

16. Hersoug, L.-G., Møller, P. \& Loft, S. Gut microbiota-derived lipopolysaccharide uptake and trafficking to adipose tissue: implications for inflammation and obesity. Obes. Rev.17 , 297-312 (2016).

17. Dehury, B. et al. Structure-Based Computational Study of Two Disease Resistance Gene Homologues (Hm1 and Hm2) in Maize (Zea mays L.) with Implications in Plant-Pathogen Interactions. PLoS One9 , e97852 (2014).

18. Toljan, K. \& Vrooman, B. Low-Dose Naltrexone (LDN)-Review of Therapeutic Utilization. Med. Sci. 6, $82(2018)$.

19. Younger, J., Parkitny, L. \& McLain, D. The use of low-dose naltrexone (LDN) as a novel antiinflammatory treatment for chronic pain. Clinical Rheumatology 33 , 451-459 (2014).

20. Hutchinson, M. R. et al. Non-stereoselective reversal of neuropathic pain by naloxone and naltrexone: Involvement of toll-like receptor 4 (TLR4). Eur. J. Neurosci. 28, 20-29 (2008).

21. Li, Z., You, Y., Griffin, N., Feng, J. \& Shan, F. Low-dose naltrexone (LDN): A promising treatment in immune-related diseases and cancer therapy. Int. Immunopharmacol. 61 , 178-184 (2018). 
22. Low-Dose Naltrexone for Pruritus in Systemic Sclerosis. Available at: https://www.ncbi.nlm.nih.gov/pmc/articles/PMC3171757/. (Accessed: 24th May 2020)

23. Lu, R. et al. Genomic characterisation and epidemiology of 2019 novel coronavirus: implications for virus origins and receptor binding. Lancet 395, 565-574 (2020).

24. Zha, L. et al. Corticosteroid treatment of patients with coronavirus disease 2019 ( $<\operatorname{scp}>$ COVID $</ \operatorname{scp}>$ -19).Med. J. Aust. 212 , 416-420 (2020).

25. Stockman, L. J., Bellamy, R. \& Garner, P. SARS: Systematic Review of Treatment Effects. PLoS Med. 3, e343 (2006).

26. Wu, F. et al. A new coronavirus associated with human respiratory disease in China. Nature $\mathbf{5 7 9}$, 265-269 (2020).

27. Zhao, M. Cytokine storm and immunomodulatory therapy in COVID-19: Role of chloroquine and anti-IL-6 monoclonal antibodies. Int. J. Antimicrob. Agents (2020). doi:10.1016/j.ijantimicag.2020.105982

28. Tanaka, T., Narazaki, M. \& Kishimoto, T. Immunotherapeutic implications of IL-6 blockade for cytokine storm. Immunotherapy8 , 959-970 (2016).

29. The Uses of Low-Dose Naltrexone in Clinical Practice | Natural Medicine Journal. Available at: https://www.naturalmedicinejournal.com/journal/2018-04/uses-low-dose-naltrexone-clinical-practice. (Accessed: 7th June 2020)

30. Gold, M. S. et al. Naltrexone, opiate addiction, and endorphins. Med. Res. Rev. 2 , 211-246 (1982).

31. Parkitny, L. \& Younger, J. Reduced Pro-Inflammatory Cytokines after Eight Weeks of Low-Dose Naltrexone for Fibromyalgia. Biomedicines5 , 16 (2017).

32. Dogra, S. et al. Zinc oxide nanoparticles attenuate hepatic steatosis development in high-fat-diet fed mice through activated AMPK signaling axis. Nanomedicine Nanotechnology, Biol. Med.17 , 210-222 (2019).

33. Morris, G. M. et al. Software news and updates AutoDock4 and AutoDockTools4: Automated docking with selective receptor flexibility.J. Comput. Chem. 30 , 2785-2791 (2009).

34. Dehury, B., Behera, S. K. \& Mahapatra, N. Structural dynamics of Casein Kinase I (CKI) from malarial parasite Plasmodium falciparum (Isolate 3D7): Insights from theoretical modelling and molecular simulations. J. Mol. Graph. Model. 71, 154-166 (2017).

35. Girdhar, K. et al. Novel insights into the dynamics behavior of glucagon-like peptide-1 receptor with its small molecule agonists.J. Biomol. Struct. Dyn. 37, 3976-3986 (2019).

36. Abraham, M. J. et al. Gromacs: High performance molecular simulations through multi-level parallelism from laptops to supercomputers. SoftwareX 1 -2 , 19-25 (2015).

37. Vanommeslaeghe, K. \& MacKerell, A. D. Automation of the CHARMM general force field (CGenFF) I: Bond perception and atom typing.J. Chem. Inf. Model. 52, 3144-3154 (2012).

\section{Hosted file}

Figure merged.pdf available at https://authorea.com/users/331891/articles/458386-naltrexonea-potential-therapeutic-candidate-for-covid-19 
A

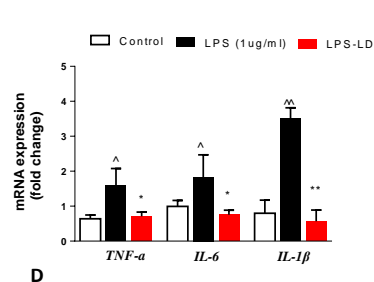

B

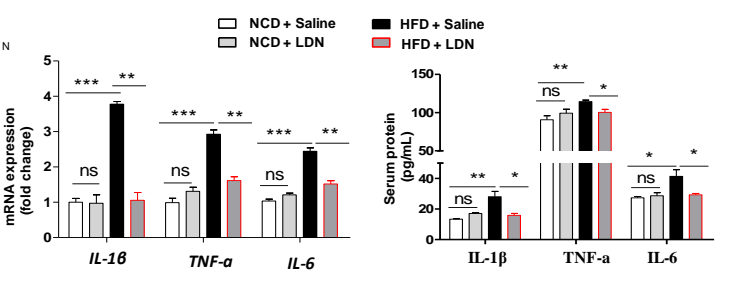

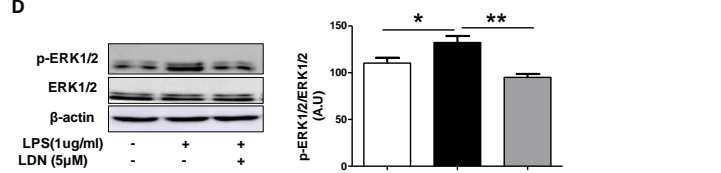

Figure 1: (A) LDN prevents LPS induced proinflammatory cytokines expression and release Quantitative mRNA expression of indicated genes (tnf- $\alpha$, il-6 and Il1b) in murine macrophage cells. (B) Quantitative mRNA expression of il-1 $\beta$, tnf- $\alpha$ and il-6 in purified ATMs from all group mice. (C) ELISA of proinflammatory proteins (IL-1 $\beta$, TNF- $\alpha$ and IL-6 ) in serum from all experimental group mice. Values are expressed as mean \pm SEM $(n=3)$ from three independent sets of repeats (mean \pm SEM $* * * p<0.001$, ** $\mathrm{p}<0.01 * \mathrm{p}<0.05$.) (D) LDN acts as EKR1 inhibitor and improved insulin sensitivity in LPS treated macrophage cells. ERK1/2 (Immunoblot) in RAW cells treated with LPS $(1 \mathrm{ug} / \mathrm{ml})$ in presence and absence of LDN. 